\title{
On resilience of connectivity in the evolution of random graphs
}

\author{
Luc Haller \\ Institute of Theoretical Computer Science \\ ETH Zürich \\ 8092 Zürich, Switzerland \\ hallerl@student.ethz.ch
}

\author{
Miloš Trujić* \\ Institute of Theoretical Computer Science \\ ETH Zürich \\ 8092 Zürich, Switzerland \\ mtrujic@inf.ethz.ch
}

Submitted: May 23, 2018; Accepted: Apr 22, 2019; Published: May 17, 2019

(c) The authors. Released under the CC BY-ND license (International 4.0).

\begin{abstract}
In this note we establish a resilience version of the classical hitting time result of Bollobás and Thomason regarding connectivity. A graph $G$ is said to be $\alpha$-resilient with respect to a monotone increasing graph property $\mathcal{P}$ if for every spanning subgraph $H \subseteq G$ satisfying $\operatorname{deg}_{H}(v) \leqslant \alpha \operatorname{deg}_{G}(v)$ for all $v \in V(G)$, the graph $G-H$ still possesses $\mathcal{P}$. Let $\left\{G_{i}\right\}$ be the random graph process, that is a process where, starting with an empty graph on $n$ vertices $G_{0}$, in each step $i \geqslant 1$ an edge $e$ is chosen uniformly at random among the missing ones and added to the graph $G_{i-1}$. We show that the random graph process is almost surely such that starting from $m \geqslant\left(\frac{1}{6}+o(1)\right) n \log n$, the largest connected component of $G_{m}$ is $\left(\frac{1}{2}-o(1)\right)$-resilient with respect to connectivity. The result is optimal in the sense that the constants $1 / 6$ in the number of edges and $1 / 2$ in the resilience cannot be improved upon. We obtain similar results for $k$-connectivity.
\end{abstract}

Mathematics Subject Classifications: 05C40, 05C 80

\section{Introduction}

Random graph theory dates back to 1959 and two seminal papers of Erdős and Rényi [12] and Gilbert [15]. By now it is a well-studied research area with applications in different fields. A more recent trend, started with a paper of Sudakov and $\mathrm{Vu}$ [29], is to study the resilience of certain properties in random graphs, which has since attracted considerable attention (e.g. [1, 2, 3, 9, 21, 27] and recent surveys [8, 28]). In other words, knowing that a random graph behaves in a certain way, the question then becomes how robust

*author was supported by grant no. 200021169242 of the Swiss National Science Foundation. 
that behaviour is with respect to modifications (e.g. deletion of the edges). Two of the most prominent examples of robustness of graphs can be seen in the results of Turán [30] and Dirac [11]. Turán's theorem determines how many edges one has to remove from a complete graph on $n$ vertices $K_{n}$ before it becomes $K_{r}$-free (global resilience), while Dirac's theorem measures how many edges touching each vertex one is allowed to remove before the graph ceases to be Hamiltonian (local resilience). The removal of all the edges incident to a vertex with minimum degree prevents the containment of any spanning structure (e.g. connectivity, Hamiltonicity, perfect matchings, etc.) giving a trivial upper bound on the global resilience in these cases. In order to study robustness of such properties it is natural to turn our attention to the local resilience.

In a more formal setting, local resilience of a graph $G$ with respect to a monotone increasing graph property $\mathcal{P}$ is defined as follows:

Definition 1 ( $\boldsymbol{\alpha}$-resilience). Let $G=(V, E)$ be a graph, $\mathcal{P}$ a monotone increasing graph property, and $\alpha \in[0,1]$ a constant. We say that $G$ is $\alpha$-resilient with respect to $\mathcal{P}$ if for every spanning subgraph $H \subseteq G$ satisfying $\operatorname{deg}_{H}(v) \leqslant \alpha \operatorname{deg}_{G}(v)$ for all $v \in V$, we have $G-H \in \mathcal{P}$.

Generally, being $\alpha$-resilient means that an adversary cannot destroy property $\mathcal{P}$ by removing an arbitrary $\alpha$-fraction of the edges incident to every vertex. In light of this, Dirac's theorem states that the complete graph $K_{n}$ is $(1 / 2)$-resilient with respect to Hamiltonicity. On the other hand, allowing the adversary to remove a bit more than a $(1 / 2)$-fraction of the edges incident to each vertex proves to be enough in order to destroy all Hamilton cycles, and even disconnect the graph. There is a vast number of important results in extremal combinatorics which study 'resilience' of the complete graph and we refer the interested reader to e.g. [10, 16, 17, 18, 19].

In this paper we show that one of the first results in random graph theory holds in a resilient fashion. A fundamental result of Erdös and Rényi [12] shows that if $m=$ $\frac{n}{2}\left(\log n+c_{n}\right)$, then with high probability $G_{n, m}$, a graph drawn uniformly at random among all graphs with $n$ vertices and $m$ edges, is connected if $c_{n} \rightarrow \infty$. Its strongest 'hitting time' version was shown much later by Bollobás and Thomason [6] and is the one which we consider here (see also Bollobás [5, Chapter 7]). For an integer $n \in \mathbb{N}$ we let $\left\{G_{i}\right\}$ define the random graph process as follows. Let $G_{0}$ be an empty graph on $n$ vertices; at every step $i \in\left\{1, \ldots,\left(\begin{array}{c}n \\ 2\end{array}\right)\right\}$ let $G_{i}$ be obtained from $G_{i-1}$ by choosing an edge $e \notin G_{i-1}$ uniformly at random and adding it to $G_{i}$. This defines a sequence of nested graphs $\left\{G_{i}\right\}_{i=0}^{N}$, where $N=\left(\begin{array}{l}n \\ 2\end{array}\right), G_{0}$ is an empty graph, and $G_{N}$ a complete graph on $n$ vertices.

A trivial necessary condition for a graph to be connected is that it has minimum degree at least one. It turns out that in the random graph process this is also sufficient. In other words, the edge $e_{i}$ that makes the last isolated vertex disappear w.h.p. also makes the graph $G_{i}$ connected. Put into a more formal setting, for a monotone increasing graph property $\mathcal{P}$ and a random graph process $\left\{G_{i}\right\}$, we define the hitting time with respect to

\footnotetext{
${ }^{1}$ We say that an event holds with high probability, w.h.p. for short, if the probability of it to hold tends to 1 as $n \rightarrow \infty$.
} 
$\mathcal{P}$, and write $\tau_{\mathcal{P}}$, as:

$$
\tau_{\mathcal{P}}=\min \left\{m \geqslant 0: G_{m} \in \mathcal{P}\right\} .
$$

One of the results of Bollobás and Thomason [6] proves that w.h.p. $\tau_{1}=\tau_{\text {conn }}$, where $\tau_{1}$ denotes the hitting times of 'having minimum degree at least one' and $\tau_{\text {conn }}$ that of 'connectivity'.

The hitting time statements about the random graph processes are in some sense the most precise results concerning random graphs one could hope for. Unsurprisingly, they are also the most difficult to analyse and only a handful of statements are known regarding some basic graph properties (such as connectivity, perfect matchings, Hamiltonicity, etc.).

Our contribution is to show that almost surely not only the graph is connected at the point $\tau_{1}$, but it is resiliently connected, that is it stays connected even after the adversary removes at most a $(1 / 2-o(1))$-fraction of the edges touching each vertex. This continues the line of research recently initiated by Nenadov, Steger, and the second author [26], and independently Montgomery [25], of studying resilience of properties in random graph processes. We further obtain similar results with respect to $k$-connectivity.

\subsection{Our results}

Our primary objective is to show the resilience of connectivity in the random graph process. Instead of dealing with the random graph process $\left\{G_{i}\right\}$ directly, one may find it more convenient to think about the Erdös-Rényi random graph $G_{n, m}$. It is a well-known fact that for every $m \in\left\{1, \ldots,\left(\begin{array}{l}n \\ 2\end{array}\right)\right\}$ the graph $G_{m}$ has the same distribution as $G_{n, m}$. Moreover, as long as $m$ is above a certain value (which is the case for us), standard connections between the model $G_{n, m}$ and the binomial random graph model $G_{n, p}{ }^{2}$ lead to direct consequences for this model as well.

Our first result shows that as long as $m$ is not too small, the largest connected component of $G_{n, m}$, which we refer to as the giant, is resilient with respect to connectivity.

Theorem 2. Let $\varepsilon>0$ be a constant and consider the random graph process $\left\{G_{i}\right\}$. Then w.h.p. for every $m \geqslant \frac{1+\varepsilon}{6} n \log n$ we have that the giant of $G_{m}$ is $(1 / 2-\varepsilon)$-resilient with respect to connectivity.

The value of $m$ is asymptotically optimal. Indeed, having $m=\frac{1-\varepsilon}{6} n \log n$ is enough for the existence of cherries 'attached' to the giant, i.e. two vertices of degree one with a common neighbour of degree three, which the adversary can easily disconnect by removing the edge from the degree three vertex which is not incident to a degree one vertex (see [24]). Additionally, the constant $1 / 2$ is optimal as we show in Proposition 16 at the end of Section 3.

The previous theorem immediately implies a hitting time result for the resilience of connectivity.

\footnotetext{
${ }^{2}$ For an integer $n$ and a function $0<p=p(n)<1$ we denote by $G_{n, p}$ the probability space of graphs on $n$ vertices where each edge is present with probability $p$ independently of other edges.
} 
Theorem 3. Let $\varepsilon>0$ be a constant. Consider the random graph process $\left\{G_{i}\right\}$ and let $\tau_{1}=\min \left\{m: \delta\left(G_{m}\right) \geqslant 1\right\}$ denote the step in which the last isolated vertex disappears. Then w.h.p. we have that $G_{\tau_{1}}$ is $(1 / 2-\varepsilon)$-resilient with respect to connectivity.

We now turn our attention to $k$-connectivity. Recall, a graph is said to be $k$-connected if the removal of at most $k-1$ vertices does not disconnect it. For the random graph process $\left\{G_{i}\right\}$, let us define

$$
\tau_{k}=\min \left\{m: \delta\left(G_{m}\right) \geqslant k\right\} \quad \text { and } \quad \tau_{k-\text { conn }}=\min \left\{m: G_{m} \text { is } k \text {-connected }\right\},
$$

denoting the point at which $\delta\left(G_{m}\right) \geqslant k$ and at which $G_{m}$ becomes $k$-connected, respectively. Erdős and Rényi [13] were the first to show that for $m=\frac{n}{2}(\log n+(k-1) \log \log n+$ $\left.c_{n}\right)$ w.h.p. $G_{n, m}$ is $k$-connected if $c_{n} \rightarrow \infty$, which also coincides with the threshold for $G_{n, m}$ to have minimum degree $k$. This result was later strengthened by Bollobás and Thomason [6] who proved the hitting time statement, that is w.h.p. $\tau_{k}=\tau_{k-\text { conn. }}$. Therefore, the trivial necessary condition for $k$-connectivity-having minimum degree at least $k$ - turns out to be sufficient as well.

In order to state our second result regarding $k$-connectivity, we need a slightly different notion of resilience. In the case of 1-connectivity (or simply connectivity) and 2-connectivity, (1/2-o(1))-resilience is not enough for the adversary to make the minimum degree of the graph drop below one and two, respectively. However, as soon as $k \geqslant 3$, the adversary would be allowed to remove an edge incident to a degree three vertex, and could easily prevent 3-connectivity. We go around this fact by slightly restricting the power of the adversary with the following definition.

Definition $4((\boldsymbol{\alpha}, \boldsymbol{k})$-resilience). Let $G=(V, E)$ be a graph, $\mathcal{P}$ a monotone increasing graph property, $\alpha \in[0,1]$ a constant, and $k \geqslant 1$ an integer. We say that $G$ is $(\alpha, k)$ resilient with respect to $\mathcal{P}$ if for every spanning subgraph $H \subseteq G$ such that $\operatorname{deg}_{H}(v) \leqslant$ $\alpha \operatorname{deg}_{G}(v)$ and $\operatorname{deg}_{G-H}(v) \geqslant k$ for all $v \in V$, we have $G-H \in \mathcal{P}$.

As observed above, the main obstruction for not having $k$-connectivity in the beginning of the process is the existence of vertices with degree smaller than $k$. However, something can still be said for such sparse(r) graphs. For an integer $k \geqslant 2$ and a graph $G$ we define the $k$-core of $G$ to be the (possibly empty) graph obtained by successively removing vertices of degree less than $k$ from $G$. With this in mind we are ready to state our second result.

Theorem 5. Let $k \geqslant 2$ be an integer, $\varepsilon>0$ a constant, and consider the random graph process $\left\{G_{i}\right\}$. Then w.h.p. for every $m \geqslant \frac{1+\varepsilon}{6} n \log n$ we have that the $k$-core of $G_{m}$ is $(1 / 2-\varepsilon, k)$-resilient with respect to $k$-connectivity.

Even though the value $m \geqslant n \log n / 6$ is optimal for connectivity, it is conceivable that the $k$-core is resilient with respect to $k$-connectivity much earlier in the process, that is as soon as it is $k$-connected, which happens roughly for $m=c n$, for some constant $c$ depending on $k$, as shown by Bollobás [4]. From a recent result of Montgomery [25] one deduces that the $k$-core is $(1 / 2-o(1))$-resilient with respect to 2 -connectivity, but only 
if the constant $k$ is much larger than two, and furthermore, no conclusions can be drawn for $k$-connectivity. We leave this as a question for further research.

Similarly as before we immediately obtain a hitting time version of the result.

Theorem 6. Let $k \geqslant 2$ be an integer and $\varepsilon>0$ a constant. Consider the random graph process $\left\{G_{i}\right\}$ and let $\tau_{k}=\min \left\{m: \delta\left(G_{m}\right) \geqslant k\right\}$ denote the step in which the last vertex of degree at most $k-1$ disappears. Then w.h.p. we have that $G_{\tau_{k}}$ is $(1 / 2-\varepsilon, k)$-resilient with respect to $k$-connectivity.

As a direct corollary we also get a statement about the classic notion of $(1 / 2-o(1))$ resilience for $k$-connectivity.

Corollary 7. Let $k \geqslant 2$ be an integer and $\varepsilon>0$ a constant. Consider the random graph process $\left\{G_{i}\right\}$ and let $\tau_{2 k-2}=\min \left\{m: \delta\left(G_{m}\right) \geqslant 2 k-2\right\}$ denote the step in which the last vertex of degree at most $2 k-3$ disappears. Then w.h.p. we have that $G_{\tau_{2 k-2}}$ is $(1 / 2-\varepsilon)$-resilient with respect to $k$-connectivity.

\section{Preliminaries}

Our graph theoretic notation follows standard textbooks (see, e.g. [7]). In particular, given a graph $G$ we denote by $V(G)$ and $E(G)$ the set of its vertices and edges, respectively, and by $v(G)$ and $e(G)$ their sizes. For subsets of vertices $X, Y \subseteq V(G), G[X]$ stands for the subgraph of $G$ induced by $X, G[X, Y]$ for the bipartite subgraph with bipartition $(X, Y)$, and $E_{G}(X, Y)$ denotes the set of edges between $X$ and $Y$ in $G$, i.e. $E_{G}(X, Y):=$ $\{\{v, w\}: v \in X, w \in Y$, and $\{v, w\} \in E(G)\}$ and $e_{G}(X, Y)$ denotes its size. For short, we write $E_{G}(X):=E_{G}(X, X)$ and $e_{G}(X)$ for its size. Furthermore, we write $N_{G}(X):=$ $\{v \in V(G): \exists u \in X$ such that $\{u, v\} \in E(G)\}$ for the neighbourhood of $X$ in $G$. Given a vertex $v \in V(G)$ we abbreviate $N_{G}(\{v\})$ to $N_{G}(v)$ and let $\operatorname{deg}_{G}(v)$ be the size of its neighbourhood, that is the degree of $v$ in $G$. We use $\delta(G)$ for the minimum degree of $G$. For $\ell \in \mathbb{N}$ and a vertex $v \in V(G)$, we define the $\ell$-neighbourhood of $v$ as the set of all vertices which lie at distance at most $\ell$ from $v$, and write $N_{G}^{\ell}(v)$, excluding $v$ itself. We omit the subscript $G$ whenever it is clear from the context which graph we refer to.

For $x, y, \varepsilon \in \mathbb{R}$, we write $x \in(1 \pm \varepsilon) y$ to denote $(1-\varepsilon) y \leqslant x \leqslant(1+\varepsilon) y$. Throughout, we use the natural $\log$ arithm $\log x=\log _{e} x$. Ceilings and floors are omitted whenever they are not essential. We make use of the standard asymptotic notation $o, \omega, O$, and $\Omega$. Lastly, we use subscripts with constants such as $C_{13}$ to indicate that $C_{13}$ is a constant with the properties as in the statement of Claim/Lemma/Proposition/Theorem 13.

We make use of the standard estimate for deviation of a binomially distributed random variable $\operatorname{Bin}(n, p)$ with parameters $n$ and $p$ from its mean (see, e.g. [14]).

Lemma 8 (Chernoff's inequality). Let $X \sim \operatorname{Bin}(n, p)$ and let $\mu:=\mathbb{E}[X]$. Then for all $\delta \in(0,1)$ :

$$
\operatorname{Pr}[X \geqslant(1+\delta) \mu] \leqslant e^{-\frac{\delta^{2} \mu}{3}}, \quad \text { and } \quad \operatorname{Pr}[X \leqslant(1-\delta) \mu] \leqslant e^{-\frac{\delta^{2} \mu}{2}} .
$$

Although our main results concern the random graph process, in the proof we heavily rely on the properties of the binomial random graph $G_{n, p}$. Next is a bound on the 
maximum number of edges certain subsets can have in a random graph $G_{n, p}$ (see $[20$, Corollary 2.3]).

Lemma 9. Let $p=p(n) \leqslant 0.99$. Then w.h.p. $G \sim G_{n, p}$ is such that every set $X \subseteq V$ satisfies

$$
\left(\begin{array}{c}
|X| \\
2
\end{array}\right) p-c|X| \sqrt{n p} \leqslant e(X) \leqslant\left(\begin{array}{c}
|X| \\
2
\end{array}\right) p+c|X| \sqrt{n p}
$$

for some absolute constant $c>0$.

The biggest obstacle in working with (very) sparse random graphs, that is for values of $p$ close to the threshold of connectivity, is that one cannot guarantee that w.h.p. even the degree of every vertex is concentrated around its expectation. Hence, the vertices whose degrees deviate significantly from the average require some special care. We introduce two classes of such vertices in the following definition.

Definition 10. For $\delta, p \in[0,1]$ and a graph $G$ with $n$ vertices, we define

$$
\begin{aligned}
\operatorname{TINY}_{p, \delta}(G) & =\left\{v \in V(G): \operatorname{deg}_{G}(v)<\delta n p\right\}, \\
\operatorname{ATYP}_{p, \delta}(G) & =\left\{v \in V(G): \operatorname{deg}_{G}(v) \notin(1 \pm \delta) n p\right\} .
\end{aligned}
$$

We refer to the vertices in $\operatorname{TINY}_{p, \delta}(G)$ as tiny and to the vertices in $\operatorname{ATYP}_{p, \delta}(G)$ as atypical.

Note that if $p$ in the above definition is roughly such that $n p$ is the average degree of a vertex in $G$, then one can think of TINY as a set of vertices whose degree is much smaller than the average, and ATYP as a set of vertices whose degree is even slightly away from the average. Let us point out that if $p \geqslant(1+\varepsilon) \log n / n$ and $\delta$ is small enough (depending on $\varepsilon$ ), then w.h.p. $G \sim G_{n, p}$ contains no tiny vertices, and similarly if $p \geqslant C \log n / n$ for large enough $C$ (depending on $\delta$ ), then w.h.p. $G \sim G_{n, p}$ contains no atypical vertices.

As their name indicates, the atypical vertices are 'rare' and do not occupy a significant fraction of the graph.

Lemma 11. For every $\delta>0$, if $p \geqslant \log n /(3 n)$, then $G \sim G_{n, p}$ w.h.p. satisfies:

$$
\left|\operatorname{ATYP}_{p, \delta}(G)\right| \leqslant n / \log n .
$$

Proof. For a fixed vertex $v \in V(G)$ by Chernoff's inequality we have

$$
\operatorname{Pr}\left[\operatorname{deg}_{G}(v) \notin(1 \pm \delta) n p\right] \leqslant 2 e^{-\delta^{2} n p / 3} \leqslant n^{-\gamma},
$$

for some $\gamma>0$. Therefore, the expected size of the set $\operatorname{ATYP}_{p, \delta}(G)$ is $n^{1-\gamma}$ and Markov's inequality shows that w.h.p. $\left|\operatorname{ATYP}_{p, \delta}(G)\right| \leqslant n / \log n$.

We briefly discuss the fact that with the previous definition at hand we could use a variant of [26, Definition 2.4] in order to show marginally stronger statements than the ones from Theorem 3 and Theorem 6. 
Definition 12. [26, Definition 2.4] Given a graph $G=(V, E)$, a graph property $\mathcal{P}$, constants $\alpha, \delta_{t}, \delta_{a} \in[0,1]$, and integers $K_{t}, K_{a} \in \mathbb{N}$, we say that $G$ is $\left(\alpha, \delta_{t}, K_{t}, \delta_{a}, K_{a}\right)$ resilient with respect to $\mathcal{P}$ if for every spanning subgraph $H \subseteq G$ such that

$$
\operatorname{deg}_{H}(v) \leqslant \begin{cases}\operatorname{deg}_{G}(v)-K_{t}, & \text { if } v \in \operatorname{TINY}_{p, \delta_{t}}(G), \\ \operatorname{deg}_{G}(v)-K_{a}, & \text { if } v \in \operatorname{ATYP}_{p, \delta_{a}}(G) \backslash \operatorname{TINY}_{p, \delta_{t}}(G) \\ \alpha \operatorname{deg}_{G}(v), & \text { otherwise, }\end{cases}
$$

for every $v \in V$, where $p=|E| /\left(\begin{array}{c}|V| \\ 2\end{array}\right)$, we have $G-H \in \mathcal{P}$.

However, just using $\left(1 / 2-o(1), \delta_{t}, 1, \delta_{a}, K_{a}\right)$-resilience for connectivity would not suffice for the following reason: for $m \leqslant(1 / 4-\varepsilon) n \log n$ w.h.p. there exists a path of length two 'attached' to the giant of $G_{m}$, which then an adversary could disconnect by removing the edge that 'attaches' it to the giant. Note that the removal of such an edge is not possible under the definition of $(1 / 2-o(1))$-resilience and hence we would need a slight modification in the definition above. Lastly, $\left(1 / 2-o(1), \delta_{t}, k, \delta_{a}, K_{a}\right)$-resilience would suffice for $k$ connectivity for every $k \geqslant 2$. In conclusion, we believe that such a 'complication' would greatly reduce the readability of our paper, unnecessarily distract the reader from the main point, and is as such not worth pursuing.

\section{The proof}

Recall that we are trying to prove that the random graph process $\left\{G_{i}\right\}$ is typically such that starting from $m \geqslant \frac{1+\varepsilon}{6} n \log n$ the giant of $G_{m}$ is resilient with respect to connectivity. Before diving into the details we give a brief outline of the ideas used.

We follow the exact path paved by the proof of [26, Proposition 3.1]. The most natural thing one could try is to show that for a fixed $m \geqslant n \log n / 6$ the giant of $G_{m}$ is w.h.p. resilient with respect to connectivity and apply a union bound over all such $m$. Unfortunately, this approach would fail as this probability is roughly $1-e^{-\alpha \cdot 2 m / n}$, for a small constant $\alpha>0$ - clearly not enough for a union bound over all values of $m$ of order $n \log n$. Instead, we group graphs into batches of size $\varepsilon n \log n$ and show that w.h.p. all graphs in a batch satisfy the property simultaneously. This allows us to apply a union bound over only a constant number of batches in order to cover all values of $m$ up to $C n \log n$. Choosing $C$ to be large enough, we may then cover the remaining values of $m$ individually, as now for each fixed one the statement holds with probability at least $1-o\left(n^{-3}\right)$.

For a cleaner exposition, we generate the graph $G_{m}$ with the help of a binomial random graph $G_{n, p}$, instead of doing it from scratch. Namely, we sample the graphs $G^{-} \sim G_{n, p_{0}}$ and $G_{n, p^{\prime}}$ where the values of $p_{0}$ and $p^{\prime}$ are such that $G^{-}$w.h.p. has at most some $m_{0}$ edges and $G^{+}=G^{-} \cup G_{n, p^{\prime}}$ has at least $(1+\varepsilon / 6) m_{0}$ edges. Taking now a permutation $\pi$ of the edges of $G_{n, p^{\prime}}$ uniformly at random we may generate each $G_{m}$ as a union of $G^{-}$ and the first $m-e\left(G^{-}\right)$edges given by $\pi$.

Crucially, the properties of the graphs $G^{-}$and $G^{+}$that we make use of are such that all graphs 'squeezed' in between them also satisfy them. The most important one 
concerns tiny and atypical vertices and states that not many of them can be 'clumped' together. Actually, an even stronger statement is true: adding an additional $\varepsilon$-fraction of random edges to $G^{-}$in order to obtain $G^{+}$does not make tiny and atypical vertices of $G^{-}$more clumped. The next lemma, which is a special case of [26, Lemma 2.6], captures this precisely.

Lemma 13. For every $\varepsilon>0$ there exist positive constants $\delta(\varepsilon)$ and $L(\varepsilon)$ such that if $p_{0} \geqslant(1+\varepsilon) \log n /(3 n)$ and $p^{\prime} \leqslant \varepsilon p_{0}$ then w.h.p. the following holds. Let $G^{-} \sim G_{n, p_{0}}$ and set $G^{+}=G^{-} \cup G_{n, p^{\prime}}$ and $p_{1}=1-\left(1-p_{0}\right)\left(1-p^{\prime}\right)$. Then:

(i) for every $v \in V\left(G^{+}\right)$we have $\left|N_{G^{+}}^{3}(v) \cap \operatorname{TINY}_{p_{0}, \delta}\left(G^{-}\right)\right| \leqslant 2$,

(ii) for every $v \in V\left(G^{+}\right)$we have $\left|N_{G^{+}}(v) \cap \operatorname{ATYP}_{p_{0}, \delta}\left(G^{-}\right)\right| \leqslant L$.

(iii) for every cycle $C \subseteq G^{+}$with $v(C)=3$, we have $\left|V(C) \cap \operatorname{TINY}_{p_{0}, \delta}\left(G^{-}\right)\right| \leqslant 1$.

Lastly, in order for the whole strategy to work in the resilience setting we keep only the edges of $G_{m}$ existing in $G^{-}$, but allow the adversary to remove edges with respect to the degrees in $G_{m}$.

Proposition 14. Let $k \geqslant 1$ be an integer. For every $\varepsilon>0$ and integer $m_{0} \geqslant \frac{1+\varepsilon}{6} n \log n$ the random graph process $\left\{G_{i}\right\}$ w.h.p. has the following property: for every integer $m_{0} \leqslant m \leqslant$ $(1+\varepsilon / 6) m_{0}$ the giant of $G_{m}$ is $(1 / 2-\varepsilon)$-resilient with respect to connectivity. Furthermore, if $k \geqslant 2$, the $k$-core of $G_{m}$ is $(1 / 2-\varepsilon, k)$-resilient with respect to $k$-connectivity.

Proof. Given $\varepsilon$ let us define $\delta=\min \left\{\varepsilon / 4, \delta_{13}(\varepsilon / 2)\right\}$ and $L=L_{13}(\varepsilon / 2)$, and let $c=c_{9}$. Take $p_{0}=(1-\varepsilon / 16) m_{0} /\left(\begin{array}{c}n \\ 2\end{array}\right), p^{\prime}=(\varepsilon / 2) p_{0}$, and let $G^{+}$be the union of two independent copies of random graphs $G^{-} \sim G_{n, p_{0}}$ and $G_{n, p^{\prime}}$. Then $G^{+}$is distributed as $G_{n, p_{1}}$, where $p_{1}=1-\left(1-p_{0}\right)\left(1-p^{\prime}\right)$. By Lemma 9 (or simply Chernoff's inequality) we have that the number of edges in $G^{-}$is w.h.p. at most $m_{0}$ and the number of edges in $G^{+}$is w.h.p. at least $(1+\varepsilon / 6) m_{0}$.

For the rest of the proof consider some $m_{0} \leqslant m \leqslant(1+\varepsilon / 6) m_{0}$. As the proof for both connectivity and $k$-connectivity (for $k \geqslant 2$ ) is identical, we treat them together and think of the graph we work with as the giant of $G_{m}$ in the former and as the $k$-core of $G_{m}$ in the latter. With this in mind, let $G \subseteq G_{m}$ be the giant of $G_{m}$, and respectively a subgraph obtained by iteratively removing all vertices of degree less that $k$ from $G_{m}$ for $k \geqslant 2$. Let $V=V(G)$ denote the vertex set of $G$, and let TINY and ATYP be sets of vertices defined as:

$$
\mathrm{TINY}:=\operatorname{TINY}_{p_{0}, \delta}\left(G^{-}\right) \cap V \quad \text { and } \quad \operatorname{ATYP}:=\operatorname{ATYP}_{p_{0}, \delta}\left(G^{-}\right) \cap V .
$$

It is a well-known fact that for $m \geqslant n \log n / 6$ the size of the giant (resp. the $k$-core) of $G_{m}$ is at least $(1-o(1)) n$ (cf. $\left.[14,22,23]\right)$. If we show that for every graph $H$ whose vertex degrees fulfil

$$
\operatorname{deg}_{H}(v) \leqslant \min \left\{(1 / 2-\varepsilon) \operatorname{deg}_{G}(v), \operatorname{deg}_{G}(v)-k\right\}
$$


the graph $G-H$ is $k$-connected, then $G$ is $(1 / 2-\varepsilon, k)$-resilient with respect to $k$ connectivity.

First, we list a series of properties that the graph $G$ satisfies, which we subsequently show are sufficient for proving the $k$-connectivity of $G-H$ :

(C1) for all $X \subseteq V$ we have $e_{G}(X) \leqslant\left(\begin{array}{c}|X| \\ 2\end{array}\right) p_{1}+c|X| \sqrt{n p_{1}}$,

(C2) for all $v \in V$ we have $\left|N_{G}^{3}(v) \cap \mathrm{TINY}\right| \leqslant 2$ and $\left|N_{G}(v) \cap \operatorname{ATYP}\right| \leqslant L$,

(C3) every cycle $C \subseteq G$ with $v(C)=3$ contains at most one vertex from TINY,

(C4) $|\mathrm{ATYP}| \leqslant \frac{n}{\log n}$.

We show that the properties hold in $G^{+}$and hence in every subgraph $G \subseteq G^{+}$. Property $(\mathrm{C} 1)$ is given by Lemma 9 applied to $G^{+}$with $p_{1}$ (as $p$ ). Properties $(\mathrm{C} 2)$ and (C3) hold by our choice of $\delta$ and $L$, and by Lemma 13 applied with $\varepsilon / 2$ (as $\varepsilon$ ), $p_{0}$ and $p^{\prime}$, since $p_{0} \geqslant(1+\varepsilon / 2) \log n /(3 n)$ and $p^{\prime} \leqslant \varepsilon p_{0}$. Lastly, (C4) holds by Lemma 11 .

Consider a graph $H$ on the same vertex set $V$ as $G$ which satisfies (1), and let $G^{\prime}:=$ $G-H$. We prove the $k$-connectivity of $G^{\prime}$ by showing that for every $S \subseteq V$ of size $|S| \leqslant k-1$, the neighbourhood of every $X \subseteq V \backslash S$ is not completely contained in $X$ in the graph $G^{\prime \prime}:=G^{\prime}[V \backslash S]$. Without loss of generality, we only consider $|X| \leqslant|V| / 2$. Assuming towards a contradiction that all edges incident to the vertices belonging to $X$ have both endpoints in $X$ gives

$$
2 e_{G^{\prime \prime}}(X)=\sum_{v \in X} \operatorname{deg}_{G^{\prime \prime}}(v)
$$

From (1) we see that all vertices $v$ in $G^{\prime}$ satisfy

$$
\operatorname{deg}_{G^{\prime}}(v) \geqslant \begin{cases}\max \left\{\left\lceil(1 / 2+\varepsilon) \operatorname{deg}_{G}(v)\right\rceil, k\right\}, & \text { if } v \in \text { TINY, } \\ (\delta / 2) n p_{0}, & \text { if } v \in \text { ATYP } \backslash \text { TINY } \\ (1 / 2+\varepsilon / 4) n p_{1}, & \text { otherwise, }\end{cases}
$$

where the last part follows from the fact that every vertex $v \in V \backslash$ ATYP has degree at least $(1-\delta) n p_{0}$ in $G$ and thus

$$
\operatorname{deg}_{G^{\prime}}(v) \geqslant(1 / 2+\varepsilon) \operatorname{deg}_{G}(v) \geqslant(1 / 2+\varepsilon)(1-\delta) n p_{0} \geqslant(1 / 2+\varepsilon / 4) n p_{1},
$$

making use of our choice of $\delta$ and $p_{1}$ in the last inequality.

We consider two cases depending on the size of $X$ : (I) $|X| \leqslant \frac{n}{1000}$ and (II) $|X|>\frac{n}{1000}$. Let us look at (I) first. The most critical point is to show that a significant part of the vertices in $X$ are actually not in TINY. The next claim establishes precisely that.

Claim 15. $|X \cap \mathrm{TINY}| \leqslant\lfloor 2|X| / 3\rfloor$. 
Proof. Observe first that there cannot exist a path of length two in the induced subgraph $G^{\prime \prime}[X \cap \mathrm{TINY}]$. Indeed, if such a path exists, as it was a part of the giant $G$ initially, it follows that there was a vertex $v \in V$ with three tiny vertices (the ones on the path) in $N_{G}^{3}(v)$ - a contradiction to (C2). Consequently, the graph $G^{\prime \prime}[X \cap \mathrm{TINY}]$ consists only of isolated vertices and edges. Let $X_{E}$ and $X_{V}$ be the partition of $X \cap$ TINY into a matching and an independent set and let

$$
\begin{aligned}
& N_{E}:=\bigcup_{\{u, v\} \in E\left(G^{\prime \prime}\left[X_{E}\right]\right)}\left(N_{G^{\prime \prime}}(u) \cup N_{G^{\prime \prime}}(v)\right) \cap(X \backslash \mathrm{TINY}) \text { and } \\
& N_{V}:=\bigcup_{u \in X_{V}} N_{G^{\prime \prime}}(u) \cap(X \backslash \mathrm{TINY}) .
\end{aligned}
$$

If $k=1$ then as every edge $\{u, v\} \in E\left(G^{\prime \prime}\left[X_{E}\right]\right)$ was initially a part of the giant $G$, we have $\left|N_{E}\right| \geqslant\left|X_{E}\right| / 2$ due to (C2) and (3). Additionally, if $k \geqslant 2$, by (3) together with properties (C2) and (C3) one easily derives (assuming $\left|X_{E}\right|>0$, otherwise $\left|N_{E}\right| \geqslant\left|X_{E}\right|$ trivially holds)

$$
\left|N_{E}\right| \geqslant(2 k-2)\left|X_{E}\right|-|S| \geqslant\left|X_{E}\right|+(2 k-3)\left|X_{E}\right|-k+1 \geqslant\left|X_{E}\right| .
$$

Similarly, as no three vertices from $X_{V}$ can have a common neighbour in $X \backslash$ TINY by (C2), we deduce $\left|N_{V}\right| \geqslant\left\lceil\left|X_{V}\right| / 2\right\rceil$. Indeed, every vertex has at least $k$ neighbours in $X \backslash$ TINY and at most $k-1$ are removed due to the removal of $S$; moreover, at most one other vertex in $X_{V}$ can share this remaining neighbour due to (C2).

Clearly now, if the number of vertices in $X \backslash$ TINY is strictly smaller than $\left|X_{E}\right| / 2+$ $\left\lceil\left|X_{V}\right| / 2\right\rceil$ by the pigeonhole principle there is a vertex $v \in X \backslash$ TINY which has at least three vertices from $X \cap \mathrm{TINY}$ in $N_{G^{\prime \prime}}^{3}(v)$, again a contradiction to $(\mathrm{C} 2)$. The claim readily follows.

The remaining vertices can be partitioned into $X \backslash$ TINY $=X_{\text {atyp }} \cup X_{\text {typ }}$ where

$$
X_{\text {atyp }}:=(\mathrm{ATYP} \backslash \mathrm{TINY}) \cap X \quad \text { and } \quad X_{\text {typ }}:=X \backslash \text { ATYP. }
$$

Next, we show that $\left|X_{\text {typ }}\right|>\left|X_{\text {atyp }}\right|$. Assume towards contradiction that this is not the case. As $n p_{0}=\omega(1)$ and $|S| \leqslant k-1$ we have

$$
\begin{aligned}
e_{G^{\prime \prime}}\left(X_{\text {atyp }}, X_{\text {typ }}\right) & \stackrel{(3)}{\geqslant}\left((\delta / 2) n p_{0}-(k-1)\right) \cdot\left|X_{\text {atyp }}\right|-2 e_{G^{\prime \prime}}\left(X_{\text {atyp }}\right)-e_{G^{\prime \prime}}\left(X_{\text {atyp }}, \text { TINY }\right) \\
& \stackrel{(\mathrm{C} 2)}{\geqslant}(\delta / 4) n p_{0} \cdot\left|X_{\text {atyp }}\right|-2 L \cdot\left|X_{\text {atyp }}\right|-2 \cdot\left|X_{\text {atyp }}\right|>L\left|X_{\text {typ }}\right|
\end{aligned}
$$

where the last inequality follows from our assumption $\left|X_{\text {atyp }}\right| \geqslant\left|X_{\text {typ }}\right|$. Thus, a simple averaging argument shows that there exists a vertex $u \in X_{\text {typ }}$ with $\operatorname{deg}_{G^{\prime \prime}}\left(u, X_{\text {atyp }}\right)>L$, which is a contradiction to $(\mathrm{C} 2)$. Together with Claim 15 we conclude $\left|X_{\text {typ }}\right| \geqslant|X| / 6$. Therefore, we have

$$
\sum_{v \in X} \operatorname{deg}_{G^{\prime \prime}}(v) \geqslant\left|X_{\mathrm{typ}}\right| \cdot \frac{1}{2} n p_{1} \geqslant \frac{|X| n p_{1}}{12}
$$


where we again make use of (3). On the other hand, by $(\mathrm{C} 1)$ we also have $2 e_{G^{\prime \prime}}(X) \leqslant$ $|X|^{2} p_{1}+2 c|X| \sqrt{n p_{1}}$, and as $|X| \leqslant n / 1000$ the assumption (2) is wrong. Thus, $N_{G^{\prime \prime}}(X) \backslash$ $X \neq \varnothing$.

We now consider case (II). By (C4), at most $n / \log n$ vertices are in ATYP, which implies that at least $(1-\varepsilon / 8)|X|$ vertices are in $X \backslash$ ATYP, as $|X|>n / 1000$. Thus, by (3) the sum of the degrees of the vertices in $X$ in $G^{\prime \prime}$ can be bounded by

$$
\sum_{v \in X} \operatorname{deg}_{G^{\prime \prime}}(v) \geqslant(1-\varepsilon / 8)|X| \cdot(1 / 2+\varepsilon / 4) n p_{1} .
$$

Combining (C1) and the bound from above with equation (2) gives

$$
(1-\varepsilon / 8)(1 / 2+\varepsilon / 4)|X| n p_{1} \leqslant 2\left(\begin{array}{c}
|X| \\
2
\end{array}\right) p_{1}+2 c|X| \sqrt{n p_{1}} \leqslant(1 / 2+\varepsilon / 16)|X| n p_{1},
$$

where the last inequality follows from the fact that $n p_{1}=\omega(1)$ and $|X| \leqslant n / 2$-again a contradiction.

In conclusion, there is no connected component of $G^{\prime \prime}$ of size at most $|V| / 2$, which completes the proof of the proposition.

Having Proposition 14 at hand we proceed to complete the proof of the main result. For convenience of the reader we restate the theorem.

Theorem 2. Let $\varepsilon>0$ be a constant and consider the random graph process $\left\{G_{i}\right\}$. Then w.h.p. for every $m \geqslant \frac{1+\varepsilon}{6} n \log n$ we have that the giant of $G_{m}$ is $(1 / 2-\varepsilon)$-resilient with respect to connectivity.

Proof. With a slightly more careful inspection, one can see that the conclusion of Proposition 14 holds with probability $1-e^{-\alpha \cdot C \log n}$ for every fixed $m \geqslant C n \log n$, some $\alpha>0$, and for sufficiently large $C$ depending on $\varepsilon$. Therefore, a union bound over all $m \geqslant C n \log n$ shows that with probability at least $1-n^{2} \cdot e^{-\alpha C \log n}=1-o(1)$, every $G_{m}$ is resilient with respect to connectivity. Note that it is also possible to draw the same conclusion from the results of Montgomery [25] and Nenadov, Steger, and the second author [26] as connectivity is a necessary condition for Hamiltonicity (even starting at $m \geqslant \frac{1+\varepsilon}{2} n \log n$ ).

For the smaller values of $m$, consider intervals of the form $\left[\frac{1+i \varepsilon}{6} n \log n, \frac{1+(i+1) \varepsilon}{6} n \log n\right)$, for $i \in\left\{1, \ldots, C_{\varepsilon}\right\}$, where $C_{\varepsilon}$ is such that the last interval contains $C n \log n$. For each fixed interval the conclusion of Proposition 14 holds with probability $1-o(1)$, thus a union bound over constantly many intervals shows that it w.h.p. holds for all intervals simultaneously. This concludes the proof.

The proof of Theorem 5 is analogous. This immediately implies Theorem 3 and Theorem 6.

We conclude by showing that if the adversary is allowed to remove slightly more than a (1/2)-fraction of the edges incident to each vertex, then w.h.p. it is possible to make the giant of $G_{m}$ disconnected. For simplicity we only show an analogue of Proposition 14. In order to capture all values of $m$ one proceeds as in the proof of Theorem 2 . 
Proposition 16. For every $\varepsilon>0$ and integer $m_{0} \geqslant \frac{1+\varepsilon}{6} n \log n$ the random graph process $\left\{G_{i}\right\}$ w.h.p. has the following property. For every integer $m_{0} \leqslant m \leqslant(1+\varepsilon / 6) m_{0}$, the giant of $G_{m}$ is not $(1 / 2+\varepsilon)$-resilient with respect to connectivity.

Proof. Given $\varepsilon$, let $\delta=\min \left\{\varepsilon / 32, \delta_{13}(\varepsilon / 2)\right\}$, and $L=L_{13}(\varepsilon / 2)$. Take $p_{0}=(1-$ $\varepsilon / 16) m_{0} /\left(\begin{array}{l}n \\ 2\end{array}\right), p^{\prime}=(\varepsilon / 2) p_{0}$, and let $G^{+}$be the union of two independent copies of random graphs $G^{-} \sim G_{n, p_{0}}$ and $G_{n, p^{\prime}}$. Then $G^{+}$is distributed as $G_{n, p_{1}}$, where $p_{1}=$ $1-\left(1-p_{0}\right)\left(1-p^{\prime}\right)$. Similarly as before, we have that the number of edges in $G^{-}$is w.h.p. at most $m_{0}$ and the number of edges in $G^{+}$is w.h.p. at least $(1+\varepsilon / 6) m_{0}$. For the rest of the proof consider some $m_{0} \leqslant m \leqslant(1+\varepsilon / 6) m_{0}$.

Let $G$ be the giant of $G_{m}$ and $V$ its vertex set. Suppose there exists a partition $V=A \cup B$ such that the following property is satisfied:

$$
\text { for all } v \in V: \operatorname{deg}_{G[A, B]}(v) \leqslant(1 / 2+\varepsilon) \operatorname{deg}_{G}(v) \text {. }
$$

Then by taking $H$ to be a subgraph consisting of all edges in $G[A, B]$ the graph $G-H$ is not connected. In the remainder we show that such a partition indeed exists.

Consider first an arbitrary equipartition $V=A^{\prime} \cup B^{\prime}$ (i.e. $A^{\prime}$ and $B^{\prime}$ differ by at most one in size). As before, let

$$
\text { TINY }:=\operatorname{TINY}_{p_{0}, \delta}\left(G^{-}\right) \cap V \quad \text { and } \quad \operatorname{ATYP}:=\operatorname{ATYP}_{p_{0}, \delta}\left(G^{-}\right) \cap V .
$$

and assume that the property (C2) holds, which follows from Lemma 13 applied with $\varepsilon / 2$ (as $\varepsilon)$. Let $D$ be defined as:

$$
D:=\left\{v \in V: \operatorname{deg}_{G\left[A^{\prime}, B^{\prime}\right]}(v)>(1 / 2+\delta) n p_{1}\right\} .
$$

Claim 17. There exists a positive constant $L^{\prime}(\varepsilon)$ such that w.h.p. for all $v \in V$ we have $\left|N_{G^{+}}(v) \cap D\right|<L^{\prime}$.

The proof follows an analogous argument as the proof of [26, Lemma 2.7].

Proof. We show that, for a sufficiently large constant $L^{\prime}=L^{\prime}(\varepsilon)$, if $T \subseteq G^{+}$is a tree with $L^{\prime} \leqslant v(T) \leqslant 2 L^{\prime}$ vertices then it contains at most $L^{\prime}-1$ vertices from $D$, which is sufficient for the claim to hold. Clearly, if for a vertex $v \in V$ there are $L^{\prime}$ vertices in $N_{G^{+}}(v) \cap D$ then taking a tree containing $v$ and every such vertex $u$ together with the edge $\{v, u\}$ contradicts the former.

Let $T \subseteq K_{n}$ be a tree with $L^{\prime} \leqslant v(T) \leqslant 2 L^{\prime}$ and $S \subseteq V(T)$ a set of size exactly $L^{\prime}$. Let $\mathcal{E}_{T}$ denote the event that $T \subseteq G^{+}$and $\mathcal{E}_{T, S}$ that every $v \in S$ satisfies $\mid N_{G^{+}\left[A^{\prime}, B^{\prime}\right]}(v) \backslash$ $V(T) \mid>(1 / 2+\delta / 2) n p_{1}$. By Chernoff's inequality we have that a fixed vertex $v \in S$ satisfies this with probability at most

$$
\operatorname{Pr}\left[\operatorname{Bin}\left(\frac{n}{2}, p_{1}\right)>(1 / 2+\delta / 2) n p_{1}\right] \leqslant e^{-\gamma n p_{1}},
$$

for some $\gamma>0$ depending on $\delta$ (and thus $\varepsilon$ ). As these events are independent for different vertices $v, u \in S$, the probability that $\mathcal{E}_{T, S}$ holds is bounded by

$$
\operatorname{Pr}\left[\mathcal{E}_{T, S}\right] \leqslant\left(e^{-\gamma n p_{1}}\right)^{L^{\prime}}
$$


Note that $\operatorname{Pr}\left[\mathcal{E}_{T}\right]=p_{1}^{v(T)-1}$. Hence, a simple union bound over all pairs $(T, S)$ shows that the probability that some $\mathcal{E}_{T} \wedge \mathcal{E}_{T, S}$ happens is at most

$$
\operatorname{Pr}\left[\bigcup_{(T, S)}\left(\mathcal{E}_{T} \wedge \mathcal{E}_{T, S}\right)\right] \leqslant \sum_{t=L^{\prime}}^{2 L^{\prime}}\left(\begin{array}{l}
n \\
t
\end{array}\right)\left(\begin{array}{c}
t \\
L^{\prime}
\end{array}\right) t^{t-2} \cdot \operatorname{Pr}\left[\mathcal{E}_{T} \wedge \mathcal{E}_{T, S}\right] \leqslant \sum_{t=L^{\prime}}^{2 L^{\prime}} n^{t} t^{3 L^{\prime}} \cdot p_{1}^{t-1} \cdot e^{-L^{\prime} \gamma n p_{1}}
$$

As $n p_{1}=\Omega(\log n)$ and $n^{t} p_{1}^{t-1} \cdot e^{-L^{\prime} \gamma n p_{1}}$ is decreasing in $p_{1}$, this finally implies

$$
\operatorname{Pr}\left[\bigcup_{(T, S)}\left(\mathcal{E}_{T} \wedge \mathcal{E}_{T, S}\right)\right]=O_{\varepsilon}\left(n \cdot(\log n)^{2 L^{\prime}} \cdot e^{-L^{\prime} \gamma n p_{1}}\right)=o(1)
$$

for $L^{\prime}$ large enough depending on $\varepsilon$.

We now construct the partition $V=A \cup B$ as follows. In the beginning set $A_{0}:=$ $A^{\prime} \backslash($ ATYP $\cup D)$ and $B_{0}:=B^{\prime} \backslash($ ATYP $\cup D)$. We sequentially add vertices to $A_{0}$ and $B_{0}$, first those of $($ ATYP $\cup D) \backslash$ TINY and then of TINY, in an arbitrary order following a simple rule: if in step $i \geqslant 1$ we have $\operatorname{deg}_{G}\left(v, A_{i-1}\right) \geqslant \operatorname{deg}_{G}\left(v, B_{i-1}\right)$ set $A_{i}:=A_{i-1} \cup\{v\}$ and $B_{i}:=B_{i-1}$; otherwise set $A_{i}:=A_{i-1}$ and $B_{i}:=B_{i-1} \cup\{v\}$. Lastly, set $A:=A_{m}$ and $B:=B_{m}$ for $m:=\mid$ ATYP $\cup D \mid$.

Note that the degree of a vertex added at step $i \geqslant 0$ in graph $G\left[A_{i}, B_{i}\right]$ can increase by at most $L+L^{\prime}+2$ until the end of the process by $(\mathrm{C} 2)$ and Claim 17 . Therefore, for every vertex $v \in V \backslash($ ATYP $\cup D)$ we have

$$
\operatorname{deg}_{G[A, B]}(v) \leqslant(1 / 2+\delta) n p_{1}+L+L^{\prime}+2 \leqslant(1 / 2+2 \delta) n p_{1} \leqslant(1 / 2+\varepsilon / 2) \operatorname{deg}_{G}(v),
$$

as $\operatorname{deg}_{G}(v) \geqslant(1-\delta) n p_{0}, p_{1} \leqslant(1+\varepsilon / 2) p_{0}$, and due to our choice of $\delta$. Similarly, for $v \in(\mathrm{ATYP} \cup D) \backslash \mathrm{TINY}$ we have

$$
\operatorname{deg}_{G[A, B]}(v) \leqslant \operatorname{deg}_{G}(v) / 2+L+L^{\prime}+2 \leqslant(1 / 2+\varepsilon / 2) \operatorname{deg}_{G}(v),
$$

as $\operatorname{deg}_{G}(v) \geqslant \delta n p_{0}$ and due to our choice of $\delta$.

Lastly, let us look at tiny vertices. If after the end of the process there is no vertex $v \in$ TINY which has $\operatorname{deg}_{G[A, B]}(v)>\operatorname{deg}_{G}(v) / 2$, we are done. Hence, assume w.l.o.g. that $v \in A$ is such a vertex. In particular, this implies that $v$ had a neighbour $u \in$ TINY added to $B$ after $v$ itself. Since $u \in B$, it implies that there exists $w \in N_{G}(u, B)$. Consequently, due to (C2), none of the neighbours of $v$ in $A$ belong to TINY and we may move $v$ to $B$ without harming the degree of any vertex significantly (it changes by at most one for non-tiny vertices, which is negligible compared to their degree). Moreover, by doing this we cannot make additional vertices $v^{\prime} \in \mathrm{TINY}$ have $\operatorname{deg}_{G[A, B]}\left(v^{\prime}\right)>\operatorname{deg}_{G}\left(v^{\prime}\right) / 2$ and thus the rearranging process eventually stops with all vertices satisfying $(\star)$. This completes the proof.

Acknowledgements. We would like to thank the anonymous reviewers for their thorough reading of our paper and helpful comments. 


\section{References}

[1] P. Allen, J. Böttcher, J. Ehrenmüller, and A. Taraz. The bandwidth theorem in sparse graphs. arXiv: 1612.00661, 2016.

[2] J. Balogh, B. Csaba, and W. Samotij. Local resilience of almost spanning trees in random graphs. Random Structures $\&$ Algorithms, 38(1-2):121-139, 2011.

[3] J. Balogh, C. Lee, and W. Samotij. Corrádi and Hajnal's theorem for sparse random graphs. Combinatorics, Probability and Computing, 21(1-2):23-55, 2012.

[4] B. Bollobás. The evolution of sparse graphs. In Graph theory and combinatorics (Cambridge, 1983), pages 35-57. Academic Press, London, 1984.

[5] B. Bollobás. Random graphs. In Modern graph theory, pages 215-252. Springer, 1998.

[6] B. Bollobás and A. Thomason. Random graphs of small order. North-Holland Mathematics Studies, 118:47-97, 1985.

[7] J. A. Bondy and U. S. R. Murty. Graph theory, volume 244 of Graduate Texts in Mathematics. Springer, New York, 2008.

[8] J. Böttcher. Large-scale structures in random graphs. Surveys in Combinatorics 2017, 440:87, 2017.

[9] J. Böttcher, Y. Kohayakawa, and A. Taraz. Almost spanning subgraphs of random graphs after adversarial edge removal. Combinatorics, Probability and Computing, 22(5):639-683, 2013.

[10] J. Böttcher, M. Schacht, and A. Taraz. Proof of the bandwidth conjecture of Bollobás and Komlós. Mathematische Annalen, 343(1):175-205, 2009.

[11] G. A. Dirac. Some theorems on abstract graphs. Proceedings of the London Mathematical Society, 3(1):69-81, 1952.

[12] P. Erdős and A. Rényi. On random graphs I. Publicationes Mathematicae Debrecen, 6:290-297, 1959.

[13] P. Erdös and A. Rényi. On the strength of connectedness of a random graph. Acta Mathematica Academiae Scientiarum Hungarica, 12(1-2):261-267, 1964.

[14] A. Frieze and M. Karoński. Introduction to random graphs. Cambridge University Press, 2015.

[15] E. N. Gilbert. Random graphs. The Annals of Mathematical Statistics, 30(4):11411144, 1959.

[16] A. Hajnal and E. Szemerédi. Proof of a conjecture of P. Erdős. Combinatorial theory and its applications, 2:601-623, 1970.

[17] J. Komlós, G. Sárközy, and E. Szemerédi. Proof of the Alon-Yuster conjecture. Discrete Mathematics, 235(1-3):255-269, 2001.

[18] J. Komlós, G. N. Sárközy, and E. Szemerédi. On the Pósa-Seymour conjecture. Journal of Graph Theory, 29(3):167-176, 1998. 
[19] M. Kouider and Z. Lonc. Covering cycles and $k$-term degree sums. Combinatorica, 16(3):407-412, 1996.

[20] M. Krivelevich and B. Sudakov. Pseudo-random graphs. More sets, graphs and numbers, pages 199-262, 2006.

[21] C. Lee and B. Sudakov. Dirac's theorem for random graphs. Random Structures 8 Algorithms, 41(3):293-305, 2012.

[22] T. Łuczak. Sparse random graphs with a given degree sequence. In Proceedings of the Symposium on Random Graphs, Poznan, pages 165-182, 1989.

[23] T. Euczak. Size and connectivity of the $k$-core of a random graph. Discrete Mathematics, 91(1):61-68, 1991.

[24] T. Euczak and A. Ruciński. Tree-matchings in graph processes. SIAM Journal on Discrete Mathematics, 4(1):107-120, 1991.

[25] R. Montgomery. Hamiltonicity in random graphs is born resilient, 2017. arXiv: 1710.00505.

[26] R. Nenadov, A. Steger, and M. Trujić. Resilience of perfect matchings and Hamiltonicity in random graph processes. Random Structures $\&$ Algorithms, 0(0), 2018.

[27] N. Škorić, A. Steger, and M. Trujić. Local resilience of an almost spanning k-cycle in random graphs. Random Structures \& Algorithms, 53(4):728-751, 2018.

[28] B. Sudakov. Robustness of graph properties. Surveys in Combinatorics 2017, 440:372, 2017.

[29] B. Sudakov and V. H. Vu. Local resilience of graphs. Random Structures \& Algorithms, 33(4):409-433, 2008.

[30] P. Turán. Eine extremalaufgabe aus der graphentheorie. Mat. Fiz. Lapok, 48(436452):61, 1941. 